\title{
Influence of duration and number of inescapable shocks on intrashock activity and subsequent interference effects
}

\author{
CHARLES R. CROWELL and D. CHRIS ANDERSON \\ University of Notre Dame, Notre Dame, Indiana 46556
}

\begin{abstract}
A three-phase investigation of the effects of duration and number of inescapable shocks with rats was conducted. In the first phase (shock treatment), separate groups were exposed either to 64 or 128 -sec shocks or to 32,64 , or 12810 -sec shocks. Measures of intrashock activity were found to be lower for the groups exposed to 64 or 128 10-sec shocks than for any other group. In the second phase (Test Day 1), half of each group was tested for interference with FR 1, locomotor escape-avoidance learning at either 24 or $168 \mathrm{~h}$ following cessation of shock treatment, using a control procedure that was designed to equate groups for exposure to test shock. The results indicated that, relative to nonshock-treated controls, at each interval only the groups previously given 64 or 128 10-sec shocks were impaired in terms of escape frequency. However, all groups given at least 64 shocks exhibited depressed intertrial responding at the $24-\mathrm{h}$, but not the $168-\mathrm{h}$, interval. In the final phase (Test Days 2-4), the control procedure for equalizing test-shock exposure was discontinued and a pattern of interference effects was observed in terms of escapeavoidance response latency that was identical to that reported for the escape frequency in Phase 2. In general, these data were viewed as indicating that duration, but not total amount of shock, was a critical determinant of behavior during inescapable shock and of the subsequent interference effect. Both effects of duration were regarded as the product of a common associative process involving the learning of immobility tendencies to shock that served to compete with later escape-avoidance responding.
\end{abstract}

It has been well documented that exposure to a treatment involving a series of inescapable shocks can result in impairment of subsequent escape-avoidance learning (Maier \& Seligman, 1976). According to one theory, this so-called interference phenomenon is believed to result exclusively from associative and motivational deficits presumed to accompany exposure to uncontrollable shock (Maier \& Seligman, 1976). It has been maintained by advocates of this view that no specific parameters of shock other than its inescapability are required to produce such deficits (Seligman, Maier, \& Solomon, 1971, p. 355). In part, this position was based upon the results of a study with dogs by Overmier and Seligman (1967, Experiment 1), which were taken to indicate that comparable interference effects resulted from various treatments differing in the duration, number, and/or rate of presentation of the inescapable shocks. More recent findings indicate, however, that interference effects are, indeed, affected

Thanks are extended to J. V. Lupo and R. Zawadski for assistance with various aspects of the present research. We gratefully acknowledge the critical reading of a previous draft of this manuscript by J. S. Brown, J. T. Cacioppo, and C. L. Cunningham. Also, special thanks are extended to Dorothy McAllister for her invaluable assistance in the preparation of this report. Requests for reprints should be sent to either author, Department of Psychology, University of Notre Dame, Notre Dame, Indiana 46556 . by such parameters of inescapable shock as its duration (Anisman, deCatanzaro, \& Remington, 1978; Glazer \& Weiss, 1976), temporal form (Crowell, Lupo, Cunningham, \& Anderson, 1978; Lawry, Lupo, Overmier, Kochevar, Hollis, \& Anderson, 1978), and type (Lawry et al., 1978).

Evidence bearing upon the influence of inescapable shock duration on interference effects in the rat, a topic of primary concern in the present report, was first provided by Glazer and Weiss (1976). These investigators found (Experiment 2) significant differences in the interference produced by exposure to 5 or 6-sec, as compared with 2-, 3-, or 4-sec, inescapable shocks despite the fact that the conditions were equated for total amount of shock through manipulation of number. Only longer shocks were found to produce what was called a "long-term" interference with escape avoidance responding that was apparent for at least a week following the cessation of treatment. To account for this duration effect, Glazer and Weiss (1976) suggested that the consequences for behavior of long and short shocks were attributable to an associative process that occurred during the inescapable shock treatment. While shocks of either long or short duration seemed to produce an initial increase in activity following shock onset, casual observations suggested that only longer shocks were accompanied by a marked immobility reaction during later portions 
of the stimulus. This depression in movement near shock offset during longer shocks was thought to provide the conditions necessary for adventitious reinforcement of intrashock immobility. This circumstance, in turn, was believed to facilitate the development of an association between the cues of shock in the longer duration condition and reduced movement. Thus, during later test shock, a form of "learned inactivity" could occur for subjects previously exposed to long shocks. Such a reaction would be expected to compete with the active escape-avoidance responding normally observed during testing.

Anisman et al. (1978) have provided important empirical support for the suppositions of Glazer and Weiss regarding the effects of inescapable shock duration on intrashock activity. In a systematic series of studies with mice, these investigators confirmed the existence of the biphasic pattern of within-shock movement thought to be associated with exposure to longer duration shock (Experiment 11). They also found that over the course of treatment, longer shocks came to result in a lower overall level of intrashock activity than did shocks of shorter duration (Experiment 8). Moreover, like Glazer and Weiss, they observed longterm interference with test performance to result only from longer duration shock (Experiment 9). And, as before, these shock-duration-related interference effects were obtained despite equivalent total shock exposure for all shock-treated subjects.

In the investigations of both Anisman et al. (1978) and Glazer and Weiss (1976), equivalent exposure of separate groups to differing durations of inescapable shock was achieved through appropriate adjustments of shock numbers. Obviously, however, such a procedure results in a confounding of any effects of these shock parameters. Therefore, it is difficult to specify adequately the possible separate and/or interacting influences of these two shock variables. Accordingly, one major purpose of the present study was to provide such information using a factorial design involving two levels of inescapable shock duration (5 and $10 \mathrm{sec}$ ) and two levels of number of inescapable shocks (64 and 128). A separate treatment involving the same total amount of shock as the 645 -sec condition but administered by means of $3210-\mathrm{sec}$ shocks also was included.

Another purpose of this study was to assess the time course of any interference effects produced by the various shock treatments. This was accomplished by testing half of the subjects in each shock-treatment condition at $24 \mathrm{~h}$ and half at $168 \mathrm{~h}$ following inescapable shock exposure. At each interval, impairment was evaluated with reference to a separate control group not exposed to shock prior to testing. Thus, the design of the present study may be represented as a 2 (duration) by 2 (number) by 2 (treatment-test interval) factorial with a separate 3210 -sec shock group and a no-shock-treatment control group at each interval.
The study involved three separable phases distinguished by different rationales, methods, and dependent measures. For purposes of exposition, these phases (Shock Treatment, Test Day 1, and Test Days 2-4) are presented separately below.

\section{SHOCK TREATMENT}

The major purpose of this phase was to expose different groups of rats to various combinations of durations and numbers of inescapable shocks. In addition, a measure of intrashock activity was obtained during presentations of inescapable shock. In this way, it was possible to determine the extent to which the activity exhibited by the various groups during shock exposure was related to levels of subsequent interference. Therefore, these procedures provided an opportunity both to confirm the suspicions of Glazer and Weiss (1976) regarding the effects of shock duration on intrashock mobility in rats as well as to extend the generality of the findings of Anisman et al. (1978, Experiment 8) that longer duration shocks produce lower overall levels of intrashock movement than do shocks of shorter duration in mice.

\section{Method}

Subjects. The same subjects served in all phases of this investigation. They were 144 naive male Sprague-Dawley rats, 90-100 days of age at the beginning of treatment. Each was housed in a separate cage, weighed and handled daily, acclimated 7-10 days in the laboratory prior to experimentation, and given free access to food and water throughout the study.

Apparatus. The apparatus for this phase was a flat acrylic platform, elevated on wooden dowels over a painted plywood base (modified from Lykken \& Rose, 1959). The platform included separate relief areas for the subject's genitals, head, and limbs. One dowel was located between the forelimb holes and one dowel was behind each hindlimb hole. Head panels were attached to this restraint platform so that they formed a V-shape that conformed roughly to the curvature of the animal's head. They were approximately $.65-.70 \mathrm{~cm}$ from either side of the rat's head and shoulders. Microswitches, activated when these panels were pressed (15-g pressure), provided a means of recording head and upper-body movement during treatment shock. A sound-deadened, lightcontrolled, ventilated refrigerator shell was used to house the apparatus.

The treatment shock source was a constant-current, tuberegulated, dc device (Campbell \& Teghtsoonian, 1958) set at $4 \mathrm{~mA}$, as measured with a $100 \mathrm{k} \Omega$ resistor substituted for the subject. Shock was interrupted 10 times/sec with a Lehigh Valley pulse stream generator (Model 1670) that produced square-wave, on-off times of $.5 \mathrm{sec}$. All treatment contingencies were controlled with solid state equipment and a laboratory computer housed in another room.

Procedure. The complete study (i.e., all phases) was run in three identical replications. Each replication consisted of eight squads of six subjects each. Across all replications, the assignment of subjects to groups was done so as to equate for weight. For the purpose of this phase, there were six groups of 24 rats each: a noshock-treatment control (NS) and groups exposed to 645 -sec (5-64), $1285-\sec (5-128), 3210-\sec (10-32), 6410-\sec (10-64)$, or 12810 -sec $(10-128)$ inescapable pulsating dc shocks. All shocks were administered in a single 2.5 -h session. At the start of this session, each subject was lightly anesthetized with a $20-40-\mathrm{sec}$ exposure to ether in a closed jar and then strapped to the treatment plat- 
form by means of an Ace bandage placed around the torso. The forelimbs were tied together to the front dowel, and the hindlimbs were separately affixed to the respective rear dowels. A polished copper electrode, lightly moistened with electrode paste, was firmly attached to the outstretched sole of each hindpaw. The first shock to all shock-treated rats began approximately $4 \mathrm{~min}$ after the restraint procedure was completed and the subject was placed into the sound-attenuation chamber. The intrashock interval for all rats was variable $( \pm 15 \mathrm{sec})$ and averaged $1 \mathrm{~min}$. All subjects, including those in Group NS, were retained in the treatment apparatus for a time equivalent to that of the most extensive treatment (i.e., the $128 \quad 10$-sec condition). The number of movements during and between shocks, or comparable nonshock periods for Group NS, was recorded for each rat.

\section{Results and Discussion}

Group NS was found to exhibit little or no movement during the treatment phase. Accordingly, data for these subjects are not presented or included in any subsequent analysis for this phase. Both the intrashock and intershock movement measures for shocktreated rats were converted to movements per second. With each measure, a mean was computed for each consecutive block of eight shock presentations or intershock periods for every subject. For purposes of exposition, both blocked activity measures were separated into four segments: Segment 1 consisted of Blocks 1-4, for which measures for all shock-treated subjects were available; Segment 2 consisted of Blocks 5-8, from which measures could be obtained only for rats given at least 64 shocks; and Segments 3 and 4, which comprised Blocks 9-12 and 13-16, respectively, and represented data only for subjects given 128 shocks. Also, for the analyses reported in this phase, a pseudovariable was created by randomly subdividing each group in half. This dummy factor represented the treatment-test interval variable that would be manipulated in the next phase of the study. It was included in the present analyses only to verify statistically that the subdivisions thus formed within each group were comparable in terms of activity measures. Since neither this factor nor its interactions proved to be significant sources of variance, the group data reported graphically were collapsed over this variable.

Activity during shock. Figure 1 shows mean movements per second during treatment shock for the subjects in each condition as a function of blocks within each segment. It is apparent that, in each segment of treatment, 10-sec shock durations were associated with lower movement levels than those promoted by 5 -sec shocks. Subjects in both shock-duration conditions exhibited decreased movement over blocks in Segment 1 , but only the 5-sec shock-duration groups continued to show decreasing activity levels throughout Segment 2. For the 5-128 group, movement levels appear to be asymptotic in Segments 3 and 4 . The decline in activity produced by exposure to 10 -sec shocks seems to be comparable for all groups and appears to be at an asymptotic level during Segments

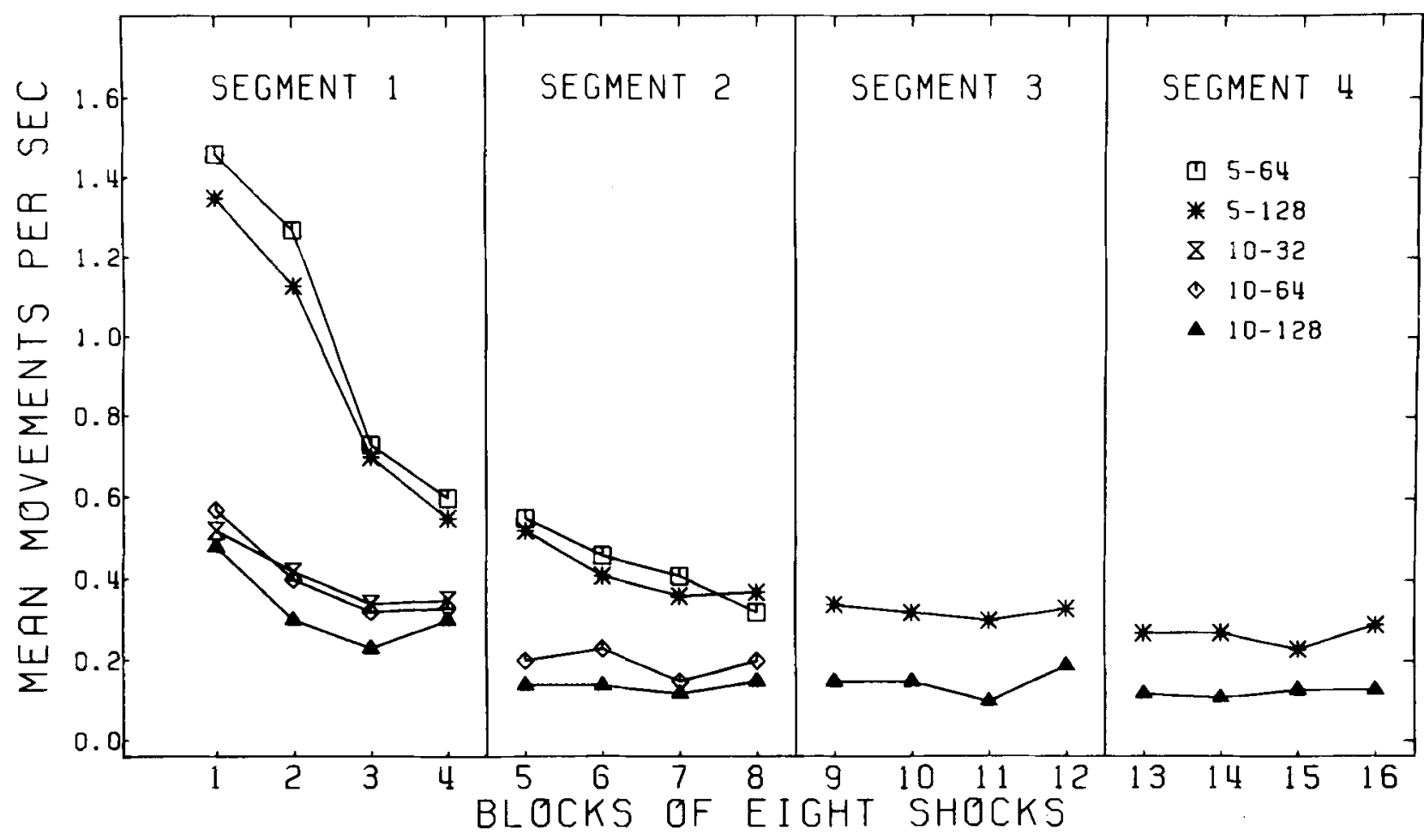

Figure 1. Mean movements per second during inescapable shock for groups exposed to 64 or 128 -sec shocks or to 32 , 64 , or 12810 -sec shocks as a function of blocks of eight shocks within each segment of treatment. 
2-4. Finally, it can be noted that even at asymptotic levels in Segments 3 and 4 , a difference in the activity levels sustained by the 1285 - and 10-sec shock-duration conditions was present.

Statistical confirmation of these visual impressions was provided by several analyses. Separate 2 (duration) by 2 (number) by 2 (dummy factor) by 4 (blocks) ANOVAs were applied to the data of Segments 1 and 2 (excluding Group 10-32), and separate 2 (duration) by 2 (dummy factor) by 4 (blocks) ANOVAs were used for Segments 3 and 4. The Segment 1 ANOVA produced significant effects only for duration $[F(1,88)$ $=66.55, \mathrm{p}<.01]$, blocks $[\mathrm{F}(3,264)=35.97, \mathrm{p}<.01]$, and Duration by Blocks $[\mathrm{F}(3,264)=15.49, \mathrm{p}<.01]$. Newman-Keuls comparisons $(\alpha=.05)$ among the groups (ignoring the dummy factor) at each block revealed that no difference within a duration condition was significant, but all differences across durations were reliable. Within-group comparisons (NewmanKeuls, $\alpha=.05$ ) of Blocks 1 and 4 for each group revealed that the decrements in activity across Segment 1 were significant for all conditions. Finally, Dunnett comparisons $(\alpha=.05)$ within each block, using a mean-square error derived from all groups, revealed that Group 10-32 did not differ from Groups 10-64 and $10-128$ but did differ from both 5 -sec groups at each point.

The Segment 2 ANOVA revealed significant effects only for duration $[\mathrm{F}(1,88)=23.09, \mathrm{p}<.01]$ and Duration by Blocks $[\mathrm{F}(3,264)=8.48, \mathrm{p}<.01]$. Newman-Keuls comparisons $(\alpha=.05)$ among the groups at each block revealed no significant differences within a duration condition, but all comparisons across duration conditions were reliable. Withingroup comparisons (Newman-Keuls, $\alpha=.05$ ) of Blocks 5 and 8 indicated that only the difference for each 5-sec duration group was significant. The ANOVAs applied to Segment 3 and 4 revealed significant effects only for duration $[\mathrm{Fs}(1,44)=15.56$ and 14.98 , respectively, ps $<.01]$.

The results of the preceding analyses were consistent in demonstrating a significant effect of shock duration in each segment. However, within any segment, number of shocks is constant for all groups, and duration is therefore confounded with total amount of shock. In order to separate the latter factors, certain a priori, cross-segment comparisons were made. On the one hand, the terminal activity levels of groups that were exposed to different shock durations but the same total amount of shock over the course of their treatments were compared. Group 1064 was found to move significantly less during shock at Block 8 than Group 5-128 at Block $16[F(1,44)=$ $12.83, \mathrm{p}<.01]$. Since both of these groups, as noted above, exhibited stable levels of intrashock activity by the end of their respective treatments, this comparison may be regarded as an indication that the long- and short-shock conditions promoted different asymptotic levels of movement despite equivalent total amounts of shock. On the other hand, the intrashock activity of Group 10-32 at Block 4 was not significantly different from that of Group 5-64 at Block 8 . This finding seems to be a reflection of the fact that 32 presentations of the 10 -sec shock was not sufficient to bring about the maximum decrease in intrashock movement that could be sustained by this duration condition. Within-group comparisons (NewmanKeuls, $\alpha=.05$ ) provided support for this interpretation by revealing significant decrements in intrashock activity from Block 4 to Block 5 for both Groups 10-64 and 10-128. Therefore, it seems reasonable to infer that the levels of within-shock activity displayed by the 10-sec groups at Block 4 were not characteristic of the lower asymptotic values subsequently exhibited under this condition.

From the foregoing it may be concluded that the different terminal asymptotic levels of intrashock activity exhibited by subjects exposed to long- and short-duration shocks in the present experiment were a function of individual shock duration per se and not of disparate total amounts of shock. This outcome both confirms and augments the previous observations of Anisman et al. (1978). One implication of this finding is that longer duration shocks may have some intrinsic property that differentiates them from shocks of shorter duration so that even a large number of exposures (i.e., 128) to the latter would not produce effects comparable to those resulting from the former. As noted, for Glazer and Weiss (1976) this special property is the biphasic intrashock activity pattern that was thought to be promoted by longer shocks and was assumed to provide for the fortuitous reinforcement of reduced activity. The possible existence of this feature of longer shocks suggests that, even from the outset of shock treatment, overall within-shock activity should be somewhat different for stimuli of long and short duration. According to this view, the presumed reinforcement mechanism would be expected to exacerbate, but not to initiate, duration-related activity differences during shock. As mentioned, evidence consistent with this notion has been provided by Anisman et al. (1978). Also, the relatively large differences between long- and short-shock groups during the first block of Segment 1 in the present experiment appears supportive of such a position. To explore this possibility further, activity in response to each of the eight shock presentations within Block 1 was examined. A 2 (duration) by 2 (number) by 2 (dummy factor) by 8 (shocks) ANOVA applied to these measures revealed a significant effect for duration $[F(1,88)=26.56, p<.01]$ but not for the Duration by Shocks interaction. These findings indicate that a difference in the intrashock activity levels promoted by long- and short-duration shocks was maintained consistently throughout the first eight shock presentations. 
Activity between shocks. Although intershock activity levels were quite low for all groups, movement rate was relatively higher for subjects exposed to the $10-\mathrm{sec}$, relative to the $5-\mathrm{sec}$, shocks, regardless of number. This effect was apparent throughout shock treatment, and no changes in movement over blocks were evident except for decreased activity in all groups across Segment 1. These observations were confirmed by the results of separate segment analyses comparable to those applied to the intrashock measure. Duration effects were significant in the ANOVAs for each segment [all $F s(1,88) \geqslant 8.40$, all ps $<.01$ ], and the blocks effect was significant in Segment 1 $[F(3,264)=5.22, p<.01]$. Dunnett tests $(\alpha=.05)$ for each block of each segment revealed that in all cases Group 10-32 did not differ from Groups 10-64 and 10-128 but did differ from both 5-sec groups. Because longer shocks resulted in more intershock movement than those of shorter duration, it is difficult to attribute the reverse pattern of effects during shock to a general loss of movement capabilities resulting from the 10-sec-duration stimuli.

\section{TEST DAY 1}

Clearly, all groups exposed to shock in the prior phase exhibited significant reductions in intrashock mobility over the course of their respective treatments. Such decreased activity may be attributable to nonassociative processes such as adaptation, fatigue, and/or shock-induced disruption of motor functioning. However, it is possible that sufficient exposure to the longer shock duration may have supplied an additional, associative mechanism that could have contributed to decreased activity during shock. This added factor could be responsible for the significantly different asymptotic intrashock activity levels characteristic of the 5-and 10 -sec conditions. Under such circumstances, exposure to different durations of inescapable shock could be expected to have different proactive consequences for subsequent escapeavoidance learning, depending upon the interval between treatment and testing.

According to the theory and evidence presented by Glazer and Weiss (1976) and Anisman et al. (1978), inactivity during prior shock that is mediated by learning can be expected to survive treatment-test intervals of varying length, thereby resulting in relatively permanent, or "long-term," interference with the subsequent acquisition of escape-avoidance behavior. Alternatively, nonassociative contributions to inactivity during shock have been characterized as being subject to dissipation within a short time following shock exposure. Test Day 1 served to evaluate these possibilities with a treatment-test interval manipulation ( 24 and $168 \mathrm{~h}$ ). If, as argued by Glazer and Weiss (1976), only relatively longer duration shocks promote the development of learned inactiv- ity, then they alone in the present study should yield perseverative impairment. Group 10-32 could be an exception, however, since this treatment apparently involved a number of shocks insufficient to promote as extensive a decrease in within-shock activity as that produced by the larger numbers of $10-\mathrm{sec}$ shocks. In fact, the terminal intrashock activity levels of this group were identical to those produced by the 5-secduration conditions that may have supplied only nonassociative sources of reduced within-shock activity during treatment. Thus, according to a learned inactivity analysis, the 10-32, 5-64, and 5-128 conditions of the present study would not be expected to produce more than a short-term debilitation of test performance. In contrast, a view of interference based upon the importance of shock uncontrollability (e.g., Maier \& Seligman, 1976) would lead to the expectation that all of the present shock treatments would lead to nondifferential interference effects, since each involved exposure to inescapable shock.

A second purpose of Test Day 1 was to compare the proactive consequences of the various shock treatments for escape-avoidance behavior and for nonshock-motivated intertrial responding during shuttle testing. Treatments that result in only a temporary disruption of motor capabilities (e.g., 5-sec shocks) should produce impairment of both types of responding, but only with relatively short treatmenttest intervals. However, treatments that promote learned inactivity (e.g., 10-64 and 10-128 conditions), as well as possible motor disruption, should result in an interference effect with both measures given a short treatment-test interval, but would be expected to produce impairment only of escape responding at a longer interval. The latter expectation derives from the suppositions that: (1) motor disruption effects will have dissipated by the time of testing with a longer interval; and (2) learned inactivity is elicited primarily by shock cues in the test situation and therefore should predominately affect behavior in the presence of shock (i.e., escape behavior).

In order to provide an accurate measure of the direct proactive effects of treatment shock upon intertrial responding during escape-avoidance testing, special restrictions must be placed upon the escapeavoidance task. The necessity for these procedures stems from the fact that if differential proactive effects of the various shock treatments occur, escape performance would be expected to vary across groups, thereby resulting in treatment-related differential exposure to test shock. If amount of test shock exposure itself were to affect intertrial responding, group differences with this measure could only be considered an indirect reflection of shock-treatment effects. To circumvent these possible difficulties, control procedures were employed that were designed to equate groups for exposure to test shock despite possible differential interference effects. 


\section{Method}

Subjects. The subjects were the same as those employed in the previous phase. In addition, two groups of 12 naive rats of the same strain and age were employed for the control purposes described below.

Apparatus. An $11.3 \mathrm{~cm}$ wide $\times 59.7 \mathrm{~cm}$ long $\times 25.0 \mathrm{~cm}$ high (i.d.) shuttlebox was employed. The shuttlebox had a floor made of grids $(.32 \mathrm{~cm}$ in diameter, spaced $1.27 \mathrm{~cm}$ apart), stainless steel walls that were connected to the grid circuit, an electrifiable cylindrical hurdle $(5.1 \mathrm{~cm}$ in diameter) mounted on ball bearings across the center of the apparatus, and 7.5-W lamps, which served as the CS, located $22 \mathrm{~cm}$ above the floor at either end of the chamber. The hurdle extended $6.3 \mathrm{~cm}$ above the floor and easily rotated when touched, thereby preventing the subject from perching on it. The grid floor was divided in half, and microswitches were attached to each floor assembly. A full crossing from one compartment to the other was required in order to activate the switches and define a response. The shock source was the scrambled, $40-\mathrm{V}$ ac output of a transformer connected in series with a $22-\mathrm{k} \Omega$ resistor. The computer recorded all data and controlled all stimulus presentations.

Procedure. Half of the subjects in each group from the previous phase were given escape-avoidance testing at $24 \mathrm{~h}$, and the other half were given escape-avoidance testing at $168 \mathrm{~h}$ following the cessation of shock treatment. The procedures used for testing subjects in this phase were designed to equate all groups for the number and duration of exposures to test shock. Methods involved in conventional escape-avoidance testing were modified in two ways to accomplish this goal. First, only three escapeavoidance trials were employed in an effort to insure that all subjects would exhibit response latencies during testing long enough to experience shock onset (i.e., to minimize the likelihood of avoidance responses). This procedure was successful inasmuch as no avoidances were observed in this phase.

The second modification involved the use of two special groups of naive rats (designated hereafter as "criterion controls") that first were exposed to the restraint-only (i.e., NS) procedure of Phase 1 and then were given three conventional escape-avoidance trials with a $5-\mathrm{sec}$ CS-US interval and a maximum trial duration of $60 \mathrm{sec}$. One of these groups was tested at $24 \mathrm{~h}$ and the other was tested at $168 \mathrm{~h}$ following the restraint-only procedure. Mean shock durations were computed from response latencies for each of these groups on each of the three trials. All experimental subjects were assigned, as an upper limit, the mean duration values obtained by the criterion groups at the appropriate treatment-test interval ( 24 or $168 \mathrm{~h}$ ) and ordinal test-trial position (i.e., 1, 2, or 3). A trial was terminated under this criterion procedure for experimental subjects whenever the criterion duration was met, or sooner if a shuttle response occurred. A 5-min interval preceded the first trial, and each intertrial interval (ITI) was 5 min. Response latencies from CS onset for each trial, three-trial total number of escapes (i.e., responses before shock offset), and intertrial responses were recorded for each rat.

\section{Results and Discussion}

The mean response latency (collapsed over trials) for the 24-h criterion control group was $18.8 \mathrm{sec}$, while that for the 168 -h group was $16.2 \mathrm{sec}$. These values are not significantly different. Since the data for these groups did not have relevance other than for establishing the upper limits of test-shock duration for this study, they are not discussed further or included in the subsequent analyses.

Response latencies. Because the criterion control procedure was used and no avoidances occurred, it was expected that exposure to test shock would be approximately equal for all groups. Therefore, re- sponse latencies should be statistically equivalent across conditions, at least within a treatment-test interval. Three sets of statistical tests were used to examine this possibility. One was provided by a 2 (duration) by 2 (number) by 2 (interval) by 3 (trials) ANOVA that produced significant effects only for interval $[F(1,88)=89.98, p<.01]$ and trials $[F(2,176)=$ $30.69, \mathrm{p}<.01]$. The interval effect resulted from a greater mean response latency at $24 \mathrm{~h}($ mean $=16.8 \mathrm{sec})$ than at $168 \mathrm{~h}($ mean $=10.9 \mathrm{sec})$. The trials effect reflected a progressive decrease in mean response latencies. A second set of tests (Dunnett, $\alpha=.05$ ) compared, within each interval, the response latencies over the three trials of each shock-treated group (including 10-32) with that of the NS control, using a meansquare error derived from all groups. The results indicated that there were no significant differences between any of the shock-treated groups and Group NS at either interval. Finally, comparisons (NewmanKeuls, $\alpha=.05$ ) within Groups $10-32$ and NS across intervals revealed significant effects comparable in both cases to the interval effect reported for the above ANOVA. Collectively, these tests indicate that the criterion control procedure produced statistically comparable exposure to test shock for all groups within an interval, but, in general, greater exposure occurred for subjects tested at the 24-h interval than for those tested at the 168-h interval. This latter finding would seem difficult to attribute to the control procedure because the criterion values across intervals were not statistically different. Also, the present influence of interval on test-shock exposure cannot be related primarily to prior effects of shock treatment because all groups within an interval, including nonshocked controls, exhibited similar effects.

Number of escapes. The mean number of escapes is displayed in the left-hand portion of Figure 2 for all groups at each treatment-test interval. It is clear

\section{ESCAPE RESPONSES INTERTRIAL RESPONSES}

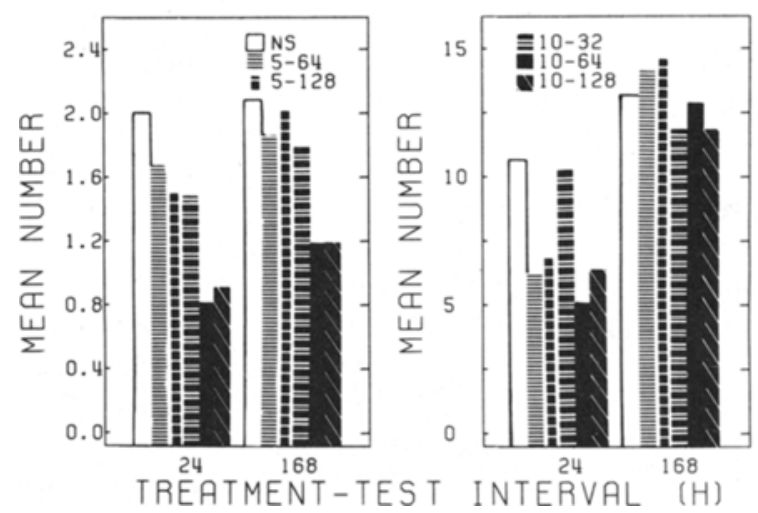

Figure 2. Mean number of escape (left) or intertrial responses (right) for all shock-treated groups and for the no-shock treatment (NS) control group at each treatment-test interval. 
that the same pattern of effects among groups was present at each interval. In both cases, only the 10-64 and 10-128 conditions resulted in a marked depression of responding relative to NS controls. However, somewhat more responding was evinced by all groups at the longer interval. A 2 (duration) by 2 (number) by 2 (interval) ANOVA produced significant effects only for duration $[F(1,88)=4.73, \mathrm{p}<.05]$ and interval $[\mathrm{F}(1,88)=16.10, \mathrm{p}<.01]$. Dunnett tests $(\alpha=$ .05) within each interval comparing each shocktreated group with the NS control indicated that, in both cases, only Groups 10-64 and 10-128 were significantly different from Group NS. Comparisons across intervals (Newman-Keuls, $\alpha=.05$ ) for Groups 10-32 and NS resulted in a significant difference only for the former group.

Thus, interference effects were obtained at both treatment-test intervals in terms of number of escapes, but only for conditions that involved at least 64 prior exposures to a $10-\mathrm{sec}$ inescapable shock. The finding of equivalent response latencies for all groups within an interval despite differential numbers of escapes is not as paradoxical as it may seem at first glance. Such a result occurred because the criterion procedure prevented the groups with few escapes (i.e., 10-64 and 10-128) from having correspondingly long mean response times. At the same time, however, the escapes that did occur during the three trials of this day were not of exceedingly short latency. Hence, comparable mean response latencies were obtained for groups displaying differential escape behavior. This finding strongly suggests that interference effects cannot be regarded as the mere byproduct of differential exposure to test shock.

Several aspects of these findings lead to the conclusion that the observed impairment on Test Day 1 should be viewed as being critically dependent upon a long-term associative process rather than as being the result merely of disrupted motor abilities. Clearly, the effects obtained at the longer interval cannot easily be attributed to a process that is commonly thought to involve only a short-lived depression of motor capability. Moreover, it is doubtful that such a mechanism could be invoked as an exclusive account of the interference observed at $24 \mathrm{~h}$, since, at both intervals, impairment was found to result from a specific duration, but not from total amount, of shock. That is, at both intervals, impairment was found in Group 10-64 but not in Group 5-128.

Nevertheless, some motor disruption may have been present at the 24-h treatment-test interval. An indication of this possibility is provided by the fact that all shock-treated groups were arithmetically somewhat impaired relative to Group NS at the shorter interval (although not significantly so, except for Groups 10-64 and 10-128). If this general depression of escape behavior were a reflection of short-term motor impairment, a significant increase in escape re- sponding from the shorter to the longer interval would be expected under all conditions except NS. Such a result was obtained.

Number of intertrial responses. The mean number of intertrial responses is depicted in the right-hand side of Figure 2 for all groups at both intervals. The pattern of results with this measure was somewhat different from that for escape frequency. At the 24-h interval, all groups except 10-32 appear to be impaired relative to Group NS, whereas no such depression is apparent for any group after $168 \mathrm{~h}$. A 2 (duration) by 2 (number) by 2 (interval) ANOVA produced significant effects only for interval $[F(1,88)$ $=45.92, \mathrm{p}<.01]$. Dunnett tests $(\alpha=.05)$ within the 24-h interval, comparing each group with NS, indicated that only the performance of 10-32 was not significantly depressed. Similar comparisons at the longer interval revealed no significant differences. Newman-Keuls $(\alpha=.05)$ comparisons across intervals within the 10-32 and NS conditions also failed to reveal any significant effects.

These results may be interpreted as indicating that the depression of intertrial, nonshock-motivated responding exhibited by all shock-treated groups, except $10-32$, at $24 \mathrm{~h}$ resulted from some form of temporary motor debilitation, inasmuch as no indication of such an effect remained at $168 \mathrm{~h}$. The latter finding also adds justification to the previous assertion that little or none of the interference with escape responding observed at $168 \mathrm{~h}$ can be accounted for in terms of a nonassociative factor. Moreover, such results further support the notion that even the impairment of escape responding obtained at $24 \mathrm{~h}$ in Groups 10-64 and 10-128 cannot be attributed exclusively to motoric disruption. This conclusion follows from the fact that duration-related interference with escape behavior was observed at the shorter interval despite nondifferential depression, except for Group 10-32, of intertrial responding. For reasons not clear, this treatment did not result at the shorter interval in the intertrial depression that was characteristic of the other shock-treated groups, including one (i.e., 5-64) that was exposed to an equivalent total amount of shock. This outcome may be an indication that impairment produced by motor debilitation, unlike associatively based interference, is dependent only upon number of treatment shocks and not upon shock duration or total amount. It must be acknowledged, however, that the differences in intertrial responding across intervals observed in this study could be at least partly due to the previously reported finding of differential test shock exposure at 24 and $168 \mathrm{~h}$. But the possible importance of this factor is minimized by the observation that intertrial responding differences across intervals were obtained for neither the 10-32 condition nor the NS condition despite differential test shock exposures at 24 and $168 \mathrm{~h}$ for both. 


\section{TEST DAYS 2-4}

The criterion control procedure used in the previous phase imposed a comparable upper limit upon the response latencies of all subjects during escapeavoidance testing. As a consequence, this strategy effectively precluded a demonstration of interference effects using the conventional response-time index. The purpose of the present phase was to determine whether or not differential impairment would be exhibited with this measure when the criterion procedure was discontinued and individual latencies were free to vary up to the maximum trial length of $60 \mathrm{sec}$. No additional manipulations, other than further escape-avoidance testing, were required for the purpose of this phase.

\section{Method}

All subjects from Phase 2, except criterion controls, were given 3 additional days of escape-avoidance testing using the previously described shuttlebox apparatus. The first day began approximately $24 \mathrm{~h}$ following the last test trial of the previous phase and consisted, for all rats, of 12 regular escape-avoidance trials with a CS-US interval of $5 \mathrm{sec}$ and a variable ITI $( \pm 30 \mathrm{sec})$ averaging $1 \mathrm{~min}$. Shock was terminated automatically on each of these trials if a response failed to occur within $60 \mathrm{sec}$ of CS onset. This procedure was repeated on each of the next 2 consecutive days.

\section{Results and Discussion}

Mean response latencies from CS onset were obtained for each subject over the 12 trials within each day. Figure 3 presents the mean daily response latencies for each group at each treatment-test interval. It is apparent that response times were not equivalent for the various groups within either interval. In each case, the most noticeable disparity occurred between the performances of Groups 10-64 and 10-128 and that of NS controls. A 2 (duration) by 2 (number) by 2 (interval) by 3 (days) ANOVA yielded significant effects only for duration $[\mathrm{F}(1,88)=6.44$, $\mathrm{p}<.05]$, interval $[\mathrm{F}(1,88)=5.23, \mathrm{p}<.05]$, and days $[F(2,176)=3.57, p<.01]$. Dunnett comparisons $(\alpha=.05)$ at each interval revealed that only Groups 10-64 and 10-128 differed significantly from Group NS. Interval effects were not found to be significant with Newman-Keuls comparisons $(\alpha=.05)$ for either the 10-32 condition or the NS condition.

The data from the present phase show that when response times were free to vary over a wider range than permitted on Test Day 1, a pattern of interference effects that was virtually identical, in terms of latency, to that observed with the escape frequency measure in the previous phase occurred. In each instance, impairment at both treatment-test intervals was found to result only from conditions involving at least 64 exposures to a 10 -sec inescapable shock. Taken together, these findings closely resemble those of Anisman et al. (1978) and Glazer and Weiss (1976) and may be viewed as a strong indication that individual shock duration is a critical determinant of proactive shock-treatment effects.

$$
24 \text { HOURS }
$$

\section{HOURS}

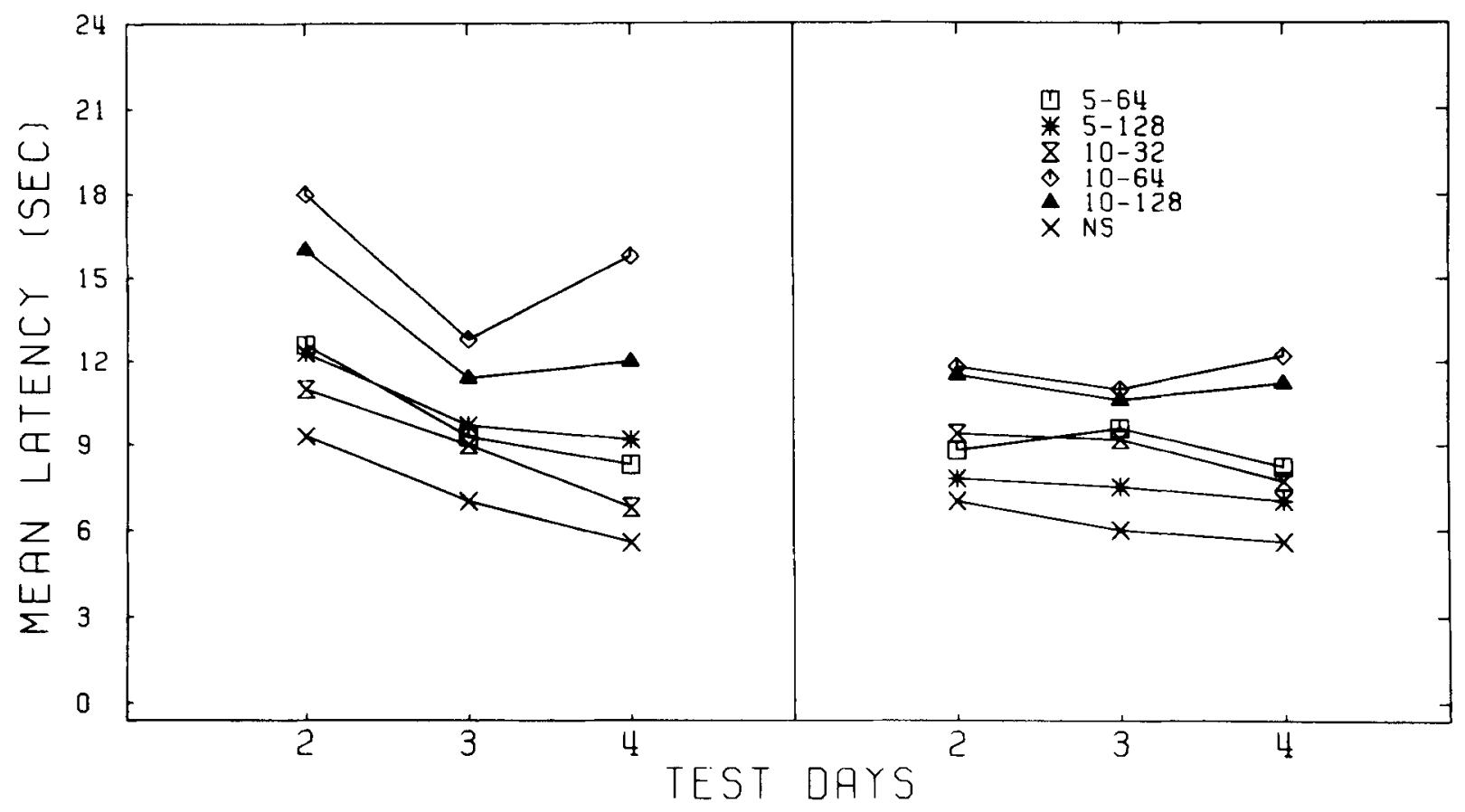

Figure 3. Mean daily escape-avoidance response latency from $\mathrm{CS}$ onset for all shock-1reated groups and for the no-shock-treatment (NS) control group at each treatment-test interval. 


\section{GENERAL DISCUSSION}

In the present investigation it was found that: (1) significant interference with escape-avoidance learning occurred at intervals of both 24 and $168 \mathrm{~h}$ following a treatment involving exposure to 64 or $12810-\mathrm{sec}$ inescapable shocks but at neither interval following only 3210 -sec or as many as 1285 -sec exposures to shock; (2) a depression of intertrial locomotor responding during testing resulted from all treatments involving at least 64 shocks, regardless of duration, at $24 \mathrm{~h}$ but from no treatment at $168 \mathrm{~h}$; and (3) those treatments that produced reliable interference also produced the lowest asymptotic levels of activity during inescapable shock.

Clearly, the present effect of shock duration cannot be attributed to any presumed consequence of shock uncontrollability, since all shock presentations in this study were programmed to be inescapable. Also, it is doubtful that duration effects resulted from confounded differences in total shock exposure. Appropriate comparisons revealed an influence of duration on activity during shock and on interference even when amount of shock was controlled. Furthermore, evidence was obtained indicating that neither duration-related activity nor interference effects were due to a general decrease in the organism's movement capabilities. If the latter were true, then the pattern of group differences when shock was present, during both treatment and testing, should have been the same as that when shock was absent (with intertrial measures), a result that was not obtained in the present study. Moreover, interference with escapeavoidance responding was found to perseverate beyond the time when motor deficits presumably should have been dissipated.

The most reasonable conclusion, on the basis of the present findings, is that the deleterious influence of inescapable shock presentations of long duration on both intrashock activity and subsequent escapeavoidance responding resulted from the learning of immobility reactions during shock. Such learning would entail the development of an association between shock stimuli and reduced activity that could result in the generalization of intrashock immobility reactions to shock cues of a subsequent test situation. Interference therein would result from the competitive influence of immobility upon the behaviors normally observed during testing. The general, if not specific, details of such an interpretation of the present results are in accord with the dictates of several recent competing response accounts of the interference phenomenon (Anderson, Crowell, Cunningham, \& Lupo, 1979; Anisman et al., 1978; Bracewell \& Black, 1974; Glazer \& Weiss, 1976).

The present data also seem congruent with the outcomes of other investigations of impairment effects resulting from exposure to inescapable presentations of dc shock (Crowell et al., 1978; Lawry et al., 1978). The latter studies were consistent in demonstrating a negative relation between levels of movement during inescapable shock and the magnitude of subsequent interference. Variations in activity during shock were produced in these experiments through manipulation of the temporal form of the inescapable shock. Exposure to 5 -sec periods of temporally continuous, inescapable dc shock was found to be accompanied by relatively low levels of intrashock activity and to produce subsequent impairment. In comparison, comparable periods of a pulsating stimulus, identical to that employed in the present study, resulted in a significantly higher level of movement during shock and little or no interference. These effects of the temporal form factor resemble those obtained in the present study with the duration variable with respect to the relation of intrashock activity to subsequent interference. In both cases, manipulations of shock parameters were found to affect within-shock movement and later impairment in ways that are consistent with a learned inactivity analysis.

However, it must be acknowledged that certain inconsistencies exist among aspects of the present findings and those of previous studies that have manipulated similar variables. For example, Glazer and Weiss (1976) found that exposure to a series of 5-sec inescapable shocks produced "long-term" interference, whereas no such effect was obtained in the present study. While this disparity may be attributed to any of several methodological differences between these studies, one of particular importance may be the qualitatively different inescapable shock stimuli used in each case. Whereas a pulsating shock was used in the present study, Glazer and Weiss (1976) apparently employed a shock of continuous form. Therefore, even though comparable in number and duration of shocks, the 5-sec treatment conditions in these studies may have differed in terms of the behaviorpromoting properties of the shock stimuli. If so, disparate proactive effects of these treatments would not be unexpected according to a competing response view.

The present results also differ from those of Anisman et al. (1978) in that the impairment effects reported herein were observed with an FR-1 shuttle procedure, whereas Anisman et al. failed to find interference with this task. The latter finding is similar to that reported by Maier, Albin, and Testa (1973), whereas the present outcome is consistent with the results of Glazer and Weiss (1976). Perhaps these seemingly disparate observations can be reconciled by noting that when interference effects were reported by Anisman et al. (1978) and Maier et al. (1973), it was in the context of a shuttle task modified (by delaying the escape reaction or requiring an FR-2 response) so as to increase the latency of initial escape responses from 1-2 sec to $4 \mathrm{sec}$ or more. According to these in- 
vestigators, such increases in initial exposure to test shock served to reduce the influence of shock-elicited locomotor activity, thereby enhancing the possibility of recording proactive interference effects. The conventional shuttle task employed herein, as well as by Glazer and Weiss, may also have served this purpose in view of the fact that NS control animals in these studies exhibited initial escape latencies well in excess of $4 \mathrm{sec}$. Possibly this was because of the relatively high hurdles and relatively low test shock intensities employed. In any case, it is clear from these investigations that interference can be obtained using a standard, FR-1 shuttle procedure.

Finally, the present findings as well as those of Anisman et al. (1978) and Glazer and Weiss (1976) differ from results reported by Overmier and Seligman (1967, Experiment 3) regarding the observed time course of shuttle impairment resulting from exposure to relatively long-duration inescapable shocks. In the former studies, interference effects were observed to persist for as long as 4-7 days following such treatment. In contrast, Overmier and Seligman found no impairment remaining after an interval of only $48 \mathrm{~h}$. How to reconcile these differences is not clear. One possibility, noted by Glazer and Weiss, is that the interference effect in the Overmier and Seligman study may have been entirely nonassociative in nature and, therefore, would not have been expected to be very persistent.

\section{REFERENCES}

Anderson, D. C., Crowell, C. R., Cunningham, C. L., \& LuPo, J. V. Behavior during shock exposure as a determinant of subsequent interference with shuttle box escape-avoidance learning in the rat. Journal of Experimental Psychology: Animal Behavior Processes, 1979, 5, 243-257.
Anisman, H., DeCatanzaro, D., \& Remington, G. Escape performance following exposure to inescapable shock: Deficits in motor response maintenance. Journal of Experimental Psychology: Animal Behavior Processes, 1978, 4, 197-218.

Brace well, R. J., \& Black, A. H. The effects of restraint and noncontingent preshock on subsequent escape learning in the rat. Learning and Motivation, 1974, 5, 53-69.

Campbell, B. A., \& Teghtsoonian, R. Electrical and behavioral effects of different types of shock stimuli on the rat. Journal of Comparative and Physiological Psychology, 1958, 51, 185-192.

Crowell, C. R., Lupo, J. V., Cunningham, C. L., \& Anderson, D. C. Temporal form of shock is a determinant of magnitude of interference with escape-avoidance learning produced by exposure to inescapable shock. Bulletin of the Psychonomic Society, 1978, 12, 407-410.

Glaze R, H. I., \& Weiss, J. M. Long-term and transitory interference effects. Journal of Experimental Psychology: Animal Behavior Processes, 1976, 2, 191-201.

Lawry, J. A., Lupo, V., Overmier, J. J., Kochevar, J., Hollis, K. L., \& ANDERSON, D. C. Interference with avoidance behavior as a function of qualitative properties of inescapable shocks. Animal Learning \& Behavior, 1978, 6, 147-154.

LYKKEn, D. T., \& Rose, R. A rat-holder with electrodes for GSR measurement. American Journal of Psychology, 1959, 72, 621-622.

Maier, S. F., Albin, R. W., \& Testa, T. J. Failure to learn to escape in rats previously exposed to inescapable shock depends on nature of escape response. Journal of Comparative and Physiological Psychology, 1973, 85, 581-592.

Maier, S. F., \& Seligman, M. E. P. Learned helplessness: Theory and evidence. Journal of Experimental Psychology: General, 1976, 105, 3-46.

Overmier, J. B., \& Seligman, M. E. P. Effects of inescapable shock upon subsequent escape and avoidance responding. Journal of Comparative and Physiological Psychology, 1967, 63, 28-33.

Seligman, M. E. P., Maier, S. F., \& Solomon, R. L. Unpredictable and uncontrollable aversive events. In F. R. Brush (Ed.), A versive conditioning and learning. New York: Academic Press, 1971.

(Received for publication March 13, 1979; revision accepted August 12, 1980.) 